\title{
REThinking lung cancer
}

\section{By Chris Cain, Staff Writer}

Less than five years after the discovery of the EML4-ALK oncogenic fusion protein as a driver of non-small cell lung cancers, and less than a year after the FDA approved Pfizer Inc.'s Xalkori crizotinib to target that fusion in these tumors, four research teams have independently identified another tyrosine kinase fusion-KIF5B-RET oncogenic fusion protein-that could underlie about $2 \%$ of lung adenocarcinoma cases. ${ }^{1-4}$ The findings provide a rationale for testing multi-tyrosine kinase inhibitors that target RET, including Sutent sunitinib, Caprelsa vandetanib and Nexavar sorafenib, in lung cancer and for developing more specific RET-targeted therapies.

One of the four groups, hailing from the Dana-Farber Cancer Institute, hopes to begin a Phase II trial of Pfizer's Sutent in KIF5B-RETpositive lung cancer patients in the first half of this year, and biotech Xcovery Inc. is now looking for more specific RET inhibitors based on the findings.

Adenocarcinoma is the most common form

\section{"These papers very nicely demonstrate the technological power we can now use to unravel genomic alterations, and [they] present a prototypical example of how smartly designed approaches can identify patients who will benefit from targeted therapy."}

\section{-Roman Thomas, University of Cologne}

of lung cancer, accounting for about $40 \%$ of cases. Many oncogenic genetic alterations have been identified in this population, including the EML4-ALK fusion and activating mutations in K-Ras and epidermal growth factor receptor (EGFR).

Identification of these alterations has led to the development of targeted therapies, the highest-profile example being Pfizer's Xalkori, a dual inhibitor of c-Met receptor tyrosine kinase (MET; c-Met; HGFR) and anaplastic lymphoma kinase (ALK), and their oncogenic variants. The drug was approved last August and is indicated for the 5\% of patients with non-small cell lung cancer (NSCLC) who have EML4ALK fusions.

However, a large fraction of adenocarcinoma patients do not carry any known oncogenic drivers. Thus, four research teams set out to identify additional genetic alterations that could inform the development of new targeted therapies for lung cancer. Each team independently came to the same conclusion: a genomic translocation that creates a fusion of kinesin family member $5 B$ (KIF5B) and ret proto-oncogene (RET) drives about $2 \%$ of lung adenocarcinoma cases.

RET is a receptor tyrosine kinase whose excess activation drives cancer cell growth. Activating mutations or translocations involving three teams tested tyrosine kinase inhibitors (TKIs) with broadspectrum activity including against RET: Caprelsa, Nexavar or Sutent. ${ }^{2-4}$ Indeed, treatment of KIF5B-RET-transformed cells with any one of the drugs inhibited growth compared with treatment using vehicle or kinase inhibitors that did not act on RET. All three compounds displayed about equivalent potency. ${ }^{4}$

Results from the Korean team were published in Genome Research, while results from the other three teams were published in Nature Medicine.

"These papers very nicely demonstrate the technological power we can now use to unravel genomic alterations, and [they] present a prototypical example of how smartly designed approaches can identify patients who will benefit from targeted therapy," said Roman Thomas, coordinator of the Clinical Lung Cancer Genome Project, a large international lung cancer genomics consortium. "This work shows us that genomic strategies have fulfilled their promise."

He noted that the genomic approaches used to identify KIF5B-RET are distinct from the functional screening approaches that led to the identification of EML4-ALK five years ago, and he thinks the new approaches will continue to lead to the identification of new cancer targets. 
Thomas, who is also professor of translational genomics at the University of Cologne, was not involved in this work. In 2010 and 2011, he published papers that found amplification of fibroblast growth factor receptor 1 (FGFR1; CD331) and mutation of discoidin domain receptor tyrosine kinase 2 (DDR2) in squamous cell lung cancer, which could lead to new targeted therapies for the disease..$^{5-8}$

Despite the different approaches, Chris Liang, CSO, EVP and director at Xcovery, said, "The RET story so far mimics the ALK story: both fusions are activating, patients with RET fusions do not have other genetic changes, and they tend to be nonsmokers. So it's tempting to conclude that inhibiting RET in these patients can have therapeutic benefits, just like the ALK inhibitor Xalkori."

\section{Finding a new purpose}

Pasi Jänne, associate professor of medicine at Harvard Medical School and Dana-Farber and colead author on the study, told SciBX that based on the results, he hopes to begin an investigator-initiated Phase II trial of Sutent in KIF5B-RET-positive lung cancer patients in "the next few months."

"I think with the data in hand, and everything we know about oncogenic kinases and kinase inhibitors, a clinical trial is a next appropriate step," said Jänne. "I don't think there is a critical piece of data that we are missing that would deter the path moving forward to the clinic. We will of course continue to study the biology of KIF5B-RET both in vitro and in vivo."

Although KIF5B-RET occurred in fewer patents than the EML4-ALK fusion, Jänne said the new findings are still important. "Lung cancer is such a common cancer that this translates into significant numbers of actual patients," he said. "With an assumption of $2 \%$, this would translate into about 4,000 new patients diagnosed annually."

Sutent is marketed to treat gastrointestinal stromal tumors and advanced renal cell carcinoma. AstraZeneca plc markets Caprelsa to treat medullary thyroid cancer. Nexavar sorafenib is marketed by Bayer AG and Onyx Pharmaceuticals Inc. for liver and renal cancers.

AstraZeneca declined to comment, and Pfizer was unavailable for comment. Onyx told SciBX that the Phase III MISSION (Monotherapy admInistration of Sorafenib in patientS with nOn-small cell luNg cancer) trial of Nexavar in NSCLC is scheduled to read out in the first half of this year, and an analysis of undisclosed biomarkers is planned for patients who elected to provide tissue samples.

"A caveat is that the reported in vitro studies employed engineered cell lines instead of patient-derived cell lines, so it is critical to clinically validate the target," said Liang.

Blueprint Medicines CSO Christoph Lengauer added that analysis of competitive kinase-binding data from the company's in-house reference database suggests the three compounds differ in how potently they inhibit RET relative to their other known clinically relevant targets. $\mathrm{He}$ told SciBX these differences may affect their clinical utility in these patients.
He said RET was the 4th, 5th and 15th most potently inhibited kinase by Caprelsa, Sorafenib and Sutent, respectively. "Given the nonselective nature of Sutent, our hypothesis is that it won't work well and that there won't be a suitable therapeutic window in patients," he said. "Caprelsa is clinically active and approved in an indication where RET is likely a relevant target, and therefore may show activity in other RET-dependent indications."

Jänne said that regardless of in vitro measurements, "at the end of the day you have to do the human experiment, which is the clinical trial." He pointed to Xalkori as an example of a drug that is not an ideal inhibitor for its ALK target but still has clear clinical benefit.

One idea to provide additional insight into the clinical effectiveness of the compounds is to analyze ongoing or prior clinical trials of the TKIs in patients with lung cancer and determine whether the KIF5B-RET fusion is correlated with clinical response. Caprelsa, Nexavar and Sutent have all been tested in NSCLC because of their broad-spectrum TKI activity.

"In all of the studies the tumor response rate to each of these agents is about $10 \%$. It would be worthwhile going back to those patient specimens to see if they contain RET rearrangements," said Jänne. "Unfortunately, the problem with many clinical trials is that they do not mandate tissue collection, and so the likelihood of such specimens being available to test is low."

Liang agreed that clinical testing of existing TKIs should begin immediately but also said there is a strong rationale to develop more specific RET inhibitors. "The available RET inhibitors have such broad-spectrum inhibitory activity that they are associated with severe toxicities at their maximum tolerated dose," he said. "More specific RET inhibitors should be better tolerated."

"We are looking into the RET activity of our compounds because of these papers," said Liang. "Our focus is to develop less-toxic compounds than the first-generation inhibitors, such as Nexavar, Sutent and Caprelsa, so that they can be more suitable for combination with other therapies."

Lengauer agreed that these findings could encourage the development of more specific RET inhibitors. "Before this work, the rationale for using drugs with RET-inhibitory activities in cancer patients was primarily based on the fact that many thyroid cancers carry mutations or translocations in RET. It likely hasn't made much sense to develop specific RET inhibitors because the number of thyroid cancer patients with RET mutations who would benefit from targeted therapy was not well understood."

He added that "because most kinase inhibitors are unselective, lots of compounds happen to hit RET, but as far as we know no one has made a truly specific RET inhibitor."

Blueprint is developing selective kinase inhibitors targeting cancers driven by genomic alterations, but the company would not comment on its pipeline. 


\section{Detection connection}

All four teams said clinical validation will go hand-in-hand with the development of diagnostics to identify patients who could respond to treatment with RET inhibitors.

Maureen Cronin, SVP of R\&D at Foundation Medicine, said the company's test is well positioned to serve that purpose. The company's sequencing test examines thousands of specific exons and introns across about 200 genes associated with cancer, including RET, at an average of more than 200-fold coverage, which Cronin said gives the test higher sensitivity and specificity than whole-transcriptome sequencing.

The company, which started collaborating with Jänne after identifying KIF5B-RET in patients with lung adenocarcinoma, has additional undisclosed collaborations looking at genomic alterations in other cancer types.

"We think, based on what we have seen so far, RET rearrangements may be found more widely in cancer outside of lung adenocarcinoma. Because of the way our test is constructed, we will be able to find all rearrangements involving RET, whereas FISH analysis could only identify specific known rearrangements," said Cronin.

The list price of the test is $\$ 5,800$, and it is performed in a CLIA lab.

In addition to Foundation Medicine, both Japanese teams are pursuing the development of undisclosed diagnostics. The Korean researchers are developing a FISH assay to detect KIF5B-RET fusions.

Foundation Medicine has filed patents covering the detection of the fusion. The National Cancer Center Research Institute also has filed for a patent covering fusion detection and is in negotiations to license it to an undisclosed diagnostic company. Macrogen Inc., which collaborated with the Korean team, has also filed for a patent and said the IP is available for licensing. JFCR did not disclose patent or licensing information.

Cain, C. SciBX 5(9); doi:10.1038/scibx.2012.219

Published online March 1, 2012

\section{REFERENCES}

1. Ju, Y.S. et al. Genome Res.; published online Dec. 22, 2011; doi:10.1101/gr.133645.111
Contact: Jeong-Sun Seo, Seoul National University, Seoul, South Korea e-mail: jeongsun@snu.ac.kr

2. Kohno, T. et al. Nat. Med.; published online Feb. 12, 2012; doi: $10.1038 / \mathrm{nm} .2644$

Contact: Takashi Kohno, National Cancer Center Research Institute, Tokyo, Japan

e-mail: tkkohno@ncc.go.jp

3. Takeuchi, K. et al. Nat. Med.; published online Feb. 12, 2012; doi: $10.1038 / \mathrm{nm} .2658$

Contact: Kengo Takeuchi, Japanese Foundation for Cancer Research, Tokyo, Japan e-mail: kentakeuchi-tky@umin.net

4. Lipson, D. et al. Nat. Med.; published online Feb. 12, 2012; doi:10.1038/nm.2673

Contact: Philip J. Stephens, Foundation Medicine Inc., Cambridge, Mass.

e-mail: pstephens@foundationmedicine.com

Contact: Maureen T. Cronin, same affiliation as above

e-mail: mcronin@foundationmedicine.com

Contact: Pasi A. Jänne, Dana-Farber Cancer Institute, Lowe Center for Thoracic Oncology, Boston, Mass.

e-mail: pjanne@partners.org

5. Weiss, J. et al. Sci. Transl. Med. 2, 62ra93 (2010)

6. Cain, C. SciBX 4(2); doi:10.1038/scibx.2011.34

7. Hammerman, P.S. et al. Cancer Discov. 1, 78-89 (2011)

8. Kotz, J. SciBX 4(20); doi:10.1038/scibx.2011.559

COMPANIES AND INSTITUTIONS MENTIONED

AstraZeneca plc (LSE:AZN; NYSE:AZN), London, U.K.

Bayer AG (Xetra:BAY), Leverkusen, Germany

Blueprint Medicines, Cambridge, Mass.

Dana-Farber Cancer Institute, Boston, Mass.

Foundation Medicine Inc., Boston, Mass.

Harvard Medical School, Boston, Mass.

Japanese Foundation for Cancer Research, Tokyo, Japan

Macrogen Inc., Seoul, South Korea

National Cancer Center Research Institute, Tokyo, Japan

Onyx Pharmaceuticals Inc. (NASDAQ:ONXX), South

San Francisco, Calif.

Pfizer Inc. (NYSE:PFE), New York, N.Y.

Seoul National University, Seoul, South Korea

University of Cologne, Cologne, Germany

Xcovery Inc., West Palm Beach, Fla. 